\title{
Calculation comparison of an additive and subtractive light modulator for high-resolution pixellight headlamps
}

\section{Marcel Philipp Held, Peer-Phillip Ley, Roland Lachmayer}

Marcel Philipp Held, Peer-Phillip Ley, Roland Lachmayer, "Calculation comparison of an additive and subtractive light modulator for high-resolution pixellight headlamps," Proc. SPIE 10554, Light-Emitting Diodes: Materials, Devices, and Applications for Solid State Lighting XXII, 1055410 (14 February 2018); doi: 10.1117/12.2290238

SPIE. Event: SPIE OPTO, 2018, San Francisco, California, United States 


\title{
Calculation comparison of an additive and subtractive light modulator for high-resolution pixellight headlamps
}

\author{
Marcel Philipp Held ${ }^{\mathrm{a}}$, Peer-Phillip Ley ${ }^{\mathrm{a}}$, and Roland Lachmayer ${ }^{\mathrm{a}}$ \\ anstitute of Product Development, Leibniz Universität Hannover, Welfengarten 1A, 30167 \\ Hanover, Germany
}

\begin{abstract}
High-resolution vehicle headlamps represent a future-oriented technology that increases traffic safety and driving comfort in the dark. A further development to current matrix beam headlamps are LED-based pixellight systems which enable additional lighting functions (e.g. the projection of navigation information on the road) to be activated for given driving scenarios.

The image generation is based on spatial light modulators (SLM) such as digital micromirror devices (DMD), liquid crystal displays (LCD), liquid crystal on silicon (LCoS) devices or LED arrays. For DMD-, LCD- and LCoSbased headlamps, the optical system uses illumining optics to ensure a precise illumination of the corresponding SLM. LED arrays, however, have to use imaging optics to project the LED die onto an intermediate image plane and thus create the light distribution via an apposition of gapless juxtapositional LED die images.

Nevertheless, the lambertian radiation characteristics complex the design of imaging optics regarding a highefficiency setup with maximum resolution and luminous flux. Simplifying the light source model and its emitting characteristics allows to determine a balanced setup between these parameters by using the Étendue and to calculate the maximum possible efficacy and luminous flux for each technology in an early designing stage.

Therefore, we present a calculation comparison of how simplifying the light source model can affect the Étendue conservation and the setup design for two high-resolution technologies. The shown approach is evaluated and compared to simulation models to show the occurring deviation and its applicability.
\end{abstract}

Keywords: Automotive Lighting, LED-based pixellight system, High-resolution headlamps, Étendue, Imaging Optics

\section{INTRODUCTION}

Increasing the resolution in vehicle headlamps offers a significant increase in traffic safety and driving comfort. First implementations of a pixel-based headlamp in automotive lighting were made by Hella with the so-called Multibeam headlamps offering 84 individual dimmable pixels. ${ }^{1}$ To provide ideal lighting functions like projecting navigation information onto the road or simultaneously dimming multiple narrow areas, the resolution still needs to be improved. ${ }^{2}$

This can be realized by using spatial light modulators (SLMs) which are responsible for the high-resolution image generation i.e. forming of the light distribution. This can be achieved by an additive modulated or an subtractive modulated approach. Subtractive light modulation can be realized for example by a digital micromirror device (DMD) which forms the light distribution by reflecting light into the optical path or onto an absorber. This concludes that driving with high beam is more efficient in these systems than driving with low beam since the light of the far-field needs to be blocked by the absorber. Additive image generators like LED arrays form the light distribution by projecting the light emitting surface of each LED gaplessly juxtapositional onto the road, apposing the light distribution by each pixel. Driving with low or high beam makes therefore no difference in the systems efficiency since the corresponding LEDs are just switched on or off.

To determine the best suitable high-resolution technology for automotive lighting applications, the Étendue can be used. Simplifying the light source model and its emitting characteristics offer the opportunity to calculate the

Further author information: (Send correspondence to M. P. Held)

M. P. Held: E-mail: held@ipeg.uni-hannover.de, Telephone: +49 5117623720

P.-P. Ley.: E-mail: ley@ipeg.uni-hannover.de, Telephone: +49 5117624591

Light-Emitting Diodes: Materials, Devices, and Applications for Solid State Lighting XXII,

edited by Jong Kyu Kim, Michael R. Krames, Martin Strassburg, Li-Wei Tu, Proc. of SPIE

Vol. 10554, 105541O - (C) 2018 SPIE · CCC code: 0277-786X/18/\$18 - doi: 10.1117/12.2290238

Proc. of SPIE Vol. 10554 1055410-1 
maximum possible efficacy and luminous flux for each technology in an early designing stage. In the following chapters, two approaches for high-resolution headlamps (DMD and LED array) will be discussed and compared to show the capability for automotive front lighting applications.

\section{SIMPLIFIED DESIGN CONFIGURATION AND EMITTER CHARACTERISTIC MODELS}

High-resolution headlamps can be categorized by the simplified design configuration shown in Figure 1 below. Typically, the semiconductor light source consists of an LED-based light source, its power supply and control unit and a corresponding primary optics (PO). The acceptance angle of the PO defines the amount of light which is guided through the optical path of the whole system. It can illuminate a SLM or image the light emitting area on an intermediate image plane (IIP). From there, the light impinges on the secondary optics (SO) and is projected onto the street. Each of the drawn elements is electrically, thermally and / or optically subject to irreversible energy losses. For this reason the main challenge in designing pixellight headlamps is to guide the

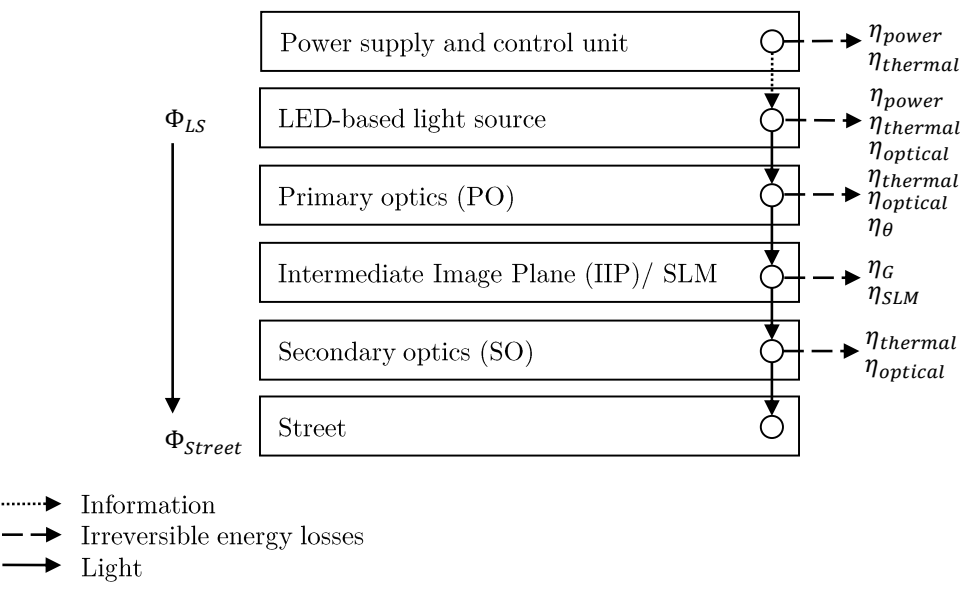

Figure 1. Simplified design configuration of high-resolution headlamps

luminous flux of the semiconductor light source $\left(\Phi_{L S}\right)$ as efficient as possible onto the street $\left(\Phi_{\text {Street }}\right)$. Most of the time, the primary optics are the main reason for a low system efficiency. ${ }^{3}$ Lambertian emitter characteristics call for big lens geometries with high acceptance angles and conflict limited installation space. This concludes automatically to a bigger magnification factor $\beta$ as can be seen in equation 1 and figure 2 .

$$
\beta=\frac{2 d^{*}}{2 l}=\frac{r^{*}}{r^{*}}
$$

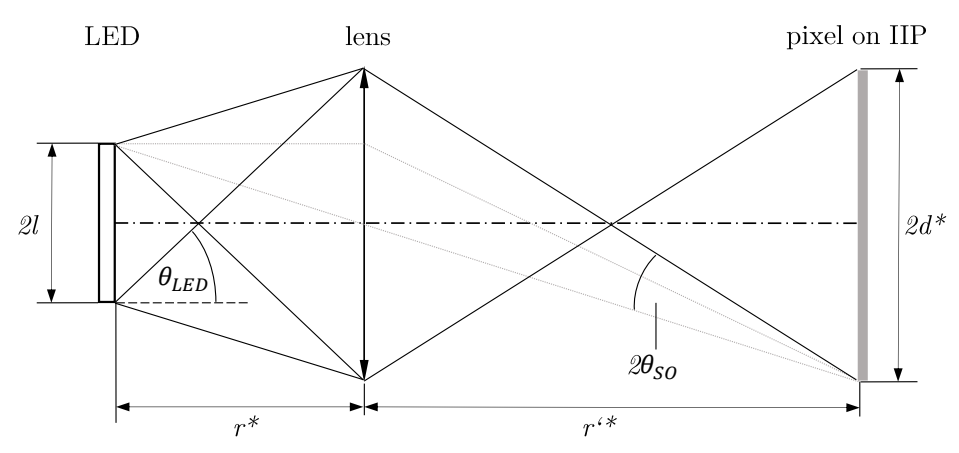

Figure 2. The theorem of intersecting lines defines the magnification factor and therefore the distances $r^{*}$ and $r^{\prime *}$ as well as the emitting and impinging angle. 
The Lagrange invariant describes the same geometric law but includes the refractive index as well as the emitting and impinging angle and is described as following:

$$
n \cdot l \cdot \theta_{L E D}=n^{\prime} \cdot d^{*} \cdot \theta_{S L M}
$$

Assuming the same refractive medium infront of and after the lens, the direct relationship between magnification factor and ratio of emitting and impinging angle can be derived:

$$
\beta=\frac{d^{*}}{l}=\frac{\sin \theta_{L E D}}{\sin \theta_{S L M}}
$$

Applying equation 2, maintaining the magnification factor follows in maintaining the acceptance angles and therefore calls for smaller lenses and distances between light source and PO, which cause higher manufacturing and positioning effort. Lowering the geometrical efficiency $\eta_{\theta}$ (which is defined by the acceptance angle) is therefore often used as a compromise to realize more compact systems:

$$
\eta_{\theta}=0.5 \cdot(1-\cos 2 \theta)
$$

With regards to figure 1, the necessary luminous flux of the light source $\Phi_{L S}$ can be calculated by

$$
\Phi_{L S}=\frac{\Phi_{\text {Street }}}{\prod \eta_{i}}
$$

To specify the necessary amount of LEDs (in other words the minimum resolution of the semiconductor light source) to emit this flux, the Étendue can be used. The Étendue $G$ is a geometrical invariant system parameter which cannot be lowered without irreversible energy losses in an ideal system but conserved or increased. It can be determined by

$$
G=N_{L E D} \cdot n \cdot \pi \cdot A \cdot \sin ^{2} \theta
$$

where $N_{L E D}$ specifies the amount of LEDs, $n$ the refractive index of the surrounding medium, $A$ the light emitting area of the semiconductor light source as well as the light emitting angle $\theta$. The Étendue of a semiconductor light source array should be smaller, best equal to the Étendue of the SLM to provide an efficient system. ${ }^{4}$ For the Étendue consideration, the emitting characteristics $\theta_{L E D}$ of an LED is simplified as shown in figure 2. Assuming an idealized characteristic of a point light source (PLS) is not sufficient for calculations due to the fact that the model does not contain information of the light source's dimensions. Thus, calculating the Étendue using equation 6 leads to an Étendue of $G_{P L S}=0$. This concludes that there is no difference in using 1 LED or 10.000 LEDs, the total efficiency as well as the total luminous flux is equal for both amount of LEDs. With regards to the emitting angle $\theta_{L E D}$ we already showed that the model assumption is suitable with engligible deviations. ${ }^{5}$ Additionally, the definition of $\theta_{L E D}$ includes a geometrical description of the LED dimensions and thus can be used for calculating the optimum system configuration:

$$
\tan \theta_{L E D}=\frac{d^{*}+l}{r^{*}}
$$

\section{3. ÉTENDUE CONSIDERATION}

In the following, a subtractive DMD-based concept and an additive LED array concept will be investigated based on a requested maximum luminous flux and efficacy. Since the least Étendue in a lighting system limits the concept's potential like a throttle does with a water flow in a ducted system, an Étendue consideration is needed. This consideration determines the ideal amount of LEDs to use in a light source array to eliminate losses caused by Étendue limits and therefore, describes the maximum efficiency and luminous flux of the concept. The following Étendue consideration is based on the V-7000 DMD from Texas Instruments and light emitting diodes WICOP-C from Seoul Semiconductors. We chose the LEDs since the ratio of LED die and housing is relatively small $(2.4: 1)$ compared to other high-power LEDs like OSRAMs BlackFlat (16:1) which allows smaller LED pitchs. An aspect ratio of $6: 1$ was estimated for the IIP of the LED-based concept. Since typical headlamp optics do not turn out to be bigger than $70 \mathrm{~mm}$ in diameter, the IIP was estimated as $60 \times 10 \mathrm{~mm}^{2}$ to still 
fit to the state-of-the-art optics. The specific parameters are listed up in Table 1. Both concepts distinguish in the light function formed by the PO (see Figure 3). The light source of a DMD-based concept typically uses PO for illumination (b), where the light of each LED is superposed onto the DMD under a limited angle of $\theta_{D M D}= \pm 12^{\circ}$. This limits the maximum luminous flux on the street by the small Étendue of the DMD. Additionally, switching single LEDs off or on generates a variation in the light intensity similar to an amplitude modulation. An LED based concept uses imaging optics to appose the light emitting surfaces on an IIP (see
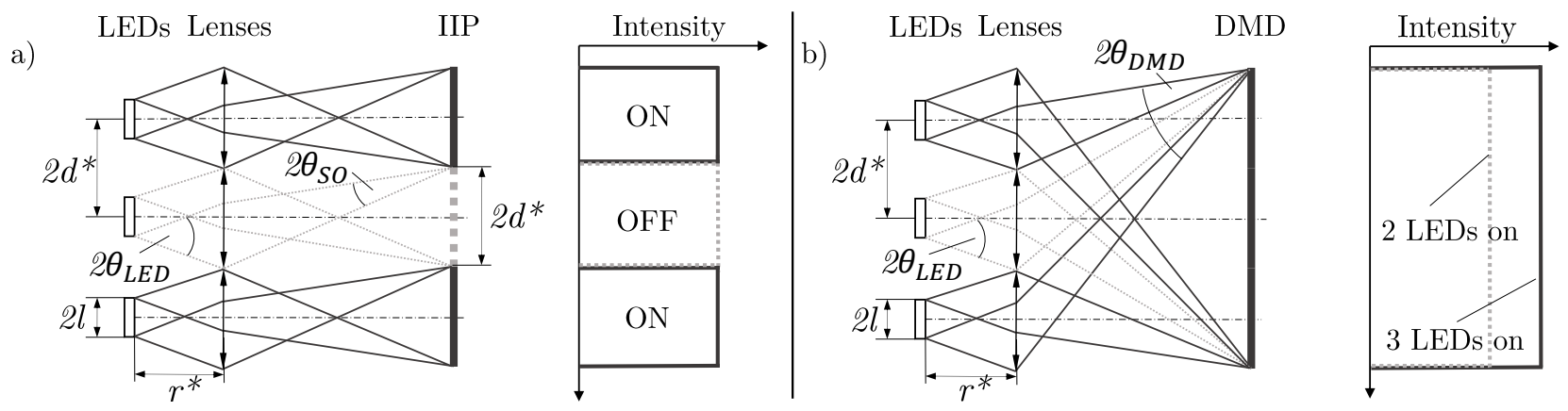

Figure 3. Appositional (a) and superpositional (b) primary optics concept

figure 3 a). In this concept the LED array modulates light by itself and thus, switching LEDs on or off causes locally dimmed pixels. The limiting factor of this concept is the impinging angle on the IIP which should not be higher than $\theta_{L E D} \leq 25^{\circ}$ to simplify the SO design. Taking the projected area as well as the impinging angle into account (which both are bigger compared to the DMD approach), this provides a higher Étendue. The Étendue of both systems can be used to determine the maximum number of LEDs i.e. luminous flux which the system is able to control efficiently by using the ratio of the spatial modulator Étendue $G_{S L M}$ and the Étendue $G_{L S}$ of the light source array. This ratio is shown in equation 8 and called Fractional Étendue: ${ }^{8}$

$$
G_{F r a c}=\frac{G_{S L M}}{G_{L S}}=\frac{A_{S L M} \cdot \sin ^{2} \theta_{D M D}}{N_{L E D} \cdot A_{L S} \cdot \sin ^{2} \theta_{L E D}} .
$$

This parameter can most likely be described as an efficiency parameter $\eta_{G}$ which specifies how well the SLM can handle the light concentration for various emitting angles $\theta_{L E D}$. This efficiency parameter can only be in the range of $0 \leq \eta_{G} \leq 1$. For the ideal case the light source Étendue equals the SLM Étendue, i.e. $\eta_{G}=G_{\text {Frac }}=1$, which should always be the aim in the design process of pixellight systems. Theoretically, the Fractional Etendue can be $G_{F r a c}>1$ which means that the limits are not reached yet and therefore the number of LEDs or their viewing angle $\theta_{L E D}$ can be increased. In this case, the efficiency $\eta_{G}$ caps the Fractional Étendue and remains constant as $\eta_{G}=1$. Vise versa, the system suffers additional irreversibilities if the Étendue is $\eta_{G}=G_{F r a c}<1$. Thus, the system efficiency of equation 5 can be described by the following:

$$
\eta_{\text {ges }}=\eta_{G} \cdot \eta_{\text {power }} \cdot \eta_{\text {thermal }} \cdot \eta_{\text {optical }} \cdot \eta_{\theta} \cdot \eta_{S L M}
$$

Presuming ideal optics and no thermal or power irreversibilities, equation 9 simplifies to

$$
\eta_{g e s}=\eta_{S L M} \cdot \eta_{\theta} \cdot \eta_{G}
$$

For this case, the parameter to highly influence the systems efficiency is the viewing angle of the LED $\theta_{L E D}$ while the parameter to influence the maximum amount of LEDs in the array is the acceptance angle of the

Table 1. Boundary conditions and datasheet extracts ${ }^{6,7}$

\begin{tabular}{lcccc}
\hline Name & Acceptance angle $\theta_{S L M}$ & $\eta_{S L M}$ & Active area $A$ & Luminous flux $\Phi_{L E D}$ \\
\hline DMD TI V-7000 & $\pm 12^{\circ}$ & 0.67 & $14.0 \times 10.5 \mathrm{~mm}^{2}$ & - \\
LED WICOP-C & - & - & $1.12 \times 1.12 \mathrm{~mm}^{2}$ & $225 \mathrm{~lm}$ \\
Image Plane IIP & $\leq \pm 25^{\circ}$ & 1 & $60 \times 10 \mathrm{~mm}^{2}$ & - \\
\hline
\end{tabular}



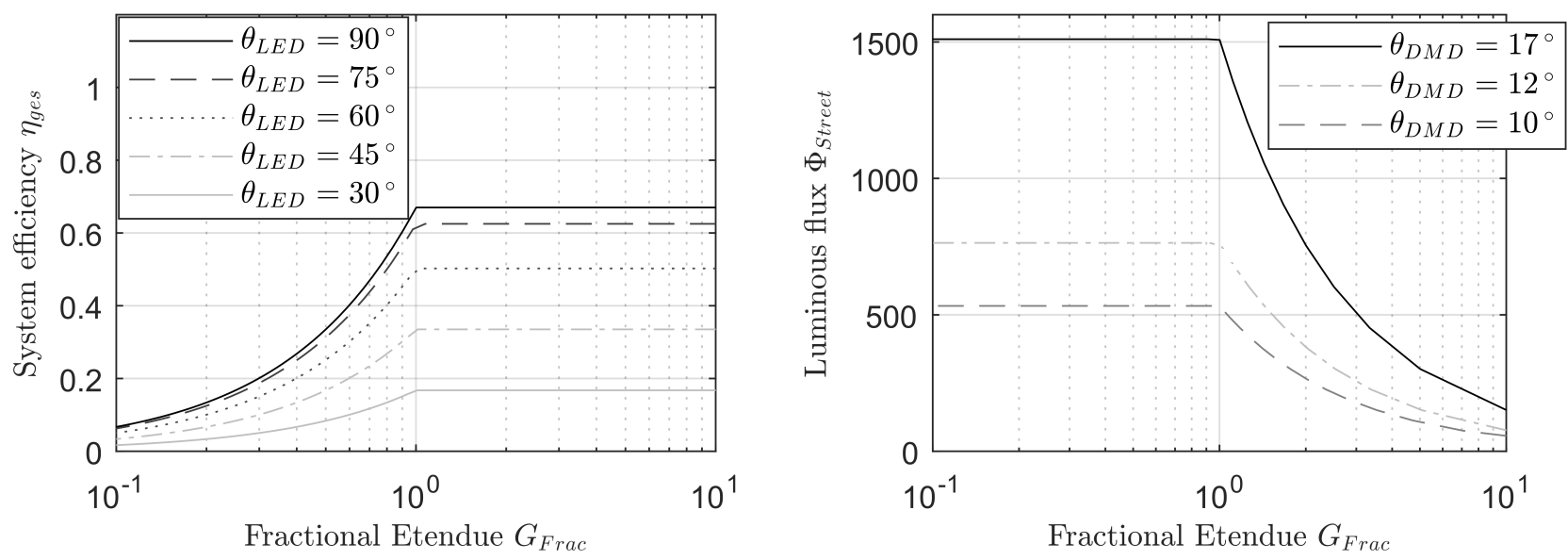

Figure 4. Fractional Étendue analysis of a DMD pixellight system

DMD $\theta_{S L M}$, the IIP respectively. Assuming the luminous flux of the LED array as the sum of the luminous flux of the single LEDs / number of LEDs $N_{L E D}$ to

$$
\Phi_{L S}=\Phi_{L E D} \cdot N_{L E D}
$$

the light source Étendue can be calculated by

$$
G_{L S}=N_{L E D} \cdot n \cdot \pi \cdot A_{L S} \cdot \sin ^{2} \theta_{L E D} .
$$

Concerning equation 8 it can be concluded that the maximum amount of LEDs, respectively pixels is mutually proportional to the Fractional Étendue:

$$
G_{F r a c} \propto \frac{1}{N_{L E D}} .
$$

The resulting luminous flux on the street $\Phi_{\text {Street }}$ can be calculated by equation 5 :

$$
\Phi_{\text {Street }}=\eta_{G} \cdot \Phi_{L S} \cdot \eta_{\theta} \cdot \eta_{S L M} .
$$

Based on this, the most suitable combination for $G_{L S}=G_{S L M}$ regarding luminous flux, efficacy and resolution can be determined. In Figure 4 the analysis for a DMD based system is shown. The whole system efficacy has been calculated for five different acceptance angles $\theta_{L E D}$ by equation 10 assuming the parameters of table 1 . It can be clearly detected that the efficacy drops significantly if the Fractional Étendue is lower than 1. Assuming the aforementioned optical, thermal and power irreversibilities as 1, the maximum possible efficacy is limited by $\eta_{D M D}$ to $\eta_{g e s}=0.67$ as shown in figure 4 . Therefore, all acceptance angles have their most efficient operating point at $G_{F r a c} \geq 1$. For each angle this concludes to different number of LEDs $N_{L E D}$. The maximum amount of luminous flux impinging on the street is limited by $\theta_{D M D}$, shown on the right side of the diagram and similar for each angle $\theta_{L E D}$. The maximum luminous flux for the TI V-7000 is therefore limited to

$$
\Phi_{\text {Street }, D M D}=763.65 \mathrm{~lm}
$$

by using $N_{L E D}=10$ LEDs at an acceptance angle of $\pm 12^{\circ}$. Presuming an LED array-modulated pixellight headlamp the potentials are much higher as shown in figure 5. Due to the acceptance angle impinging on the IIP, the realizable luminous flux is crucially higher. Furthermore, the IIP doesn't have an optical array efficiency $\eta_{S L M}$. This concludes to a maximal possible efficancy advantage of $+49 \%$ compared to DMD-based systems. Depending on which impinging angle $\theta_{I I P}$ is chosen, the maximum luminous flux output varies. Since an impinging angle up to $\theta_{I I P} \leq 25^{\circ}$ is possible, the maximum luminous flux assuming an acceptance angle of $\theta_{L E D}=90^{\circ}$ (TIR-optics, $\left.\eta_{\text {ges }}=1\right)$ is limited to

$$
\Phi_{\text {Street }, I I P}=19222 \mathrm{~lm} .
$$



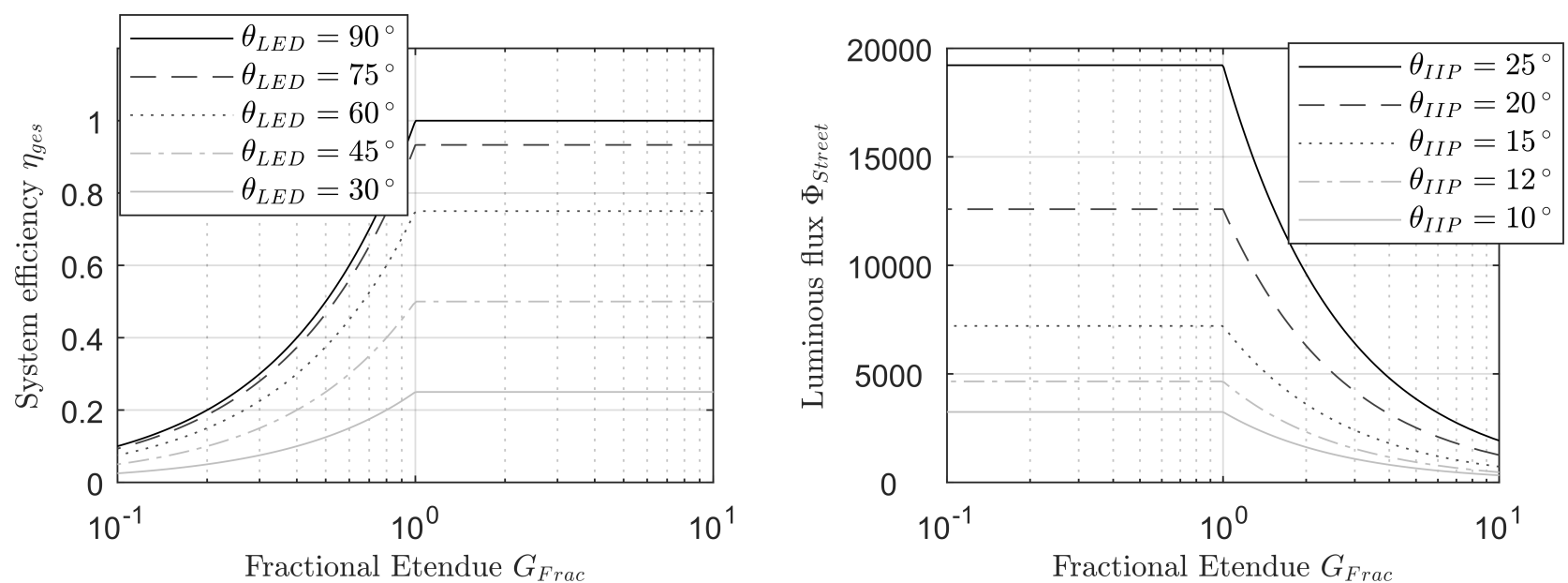

Figure 5. Fractional Étendue analysis of an LED pixellight system
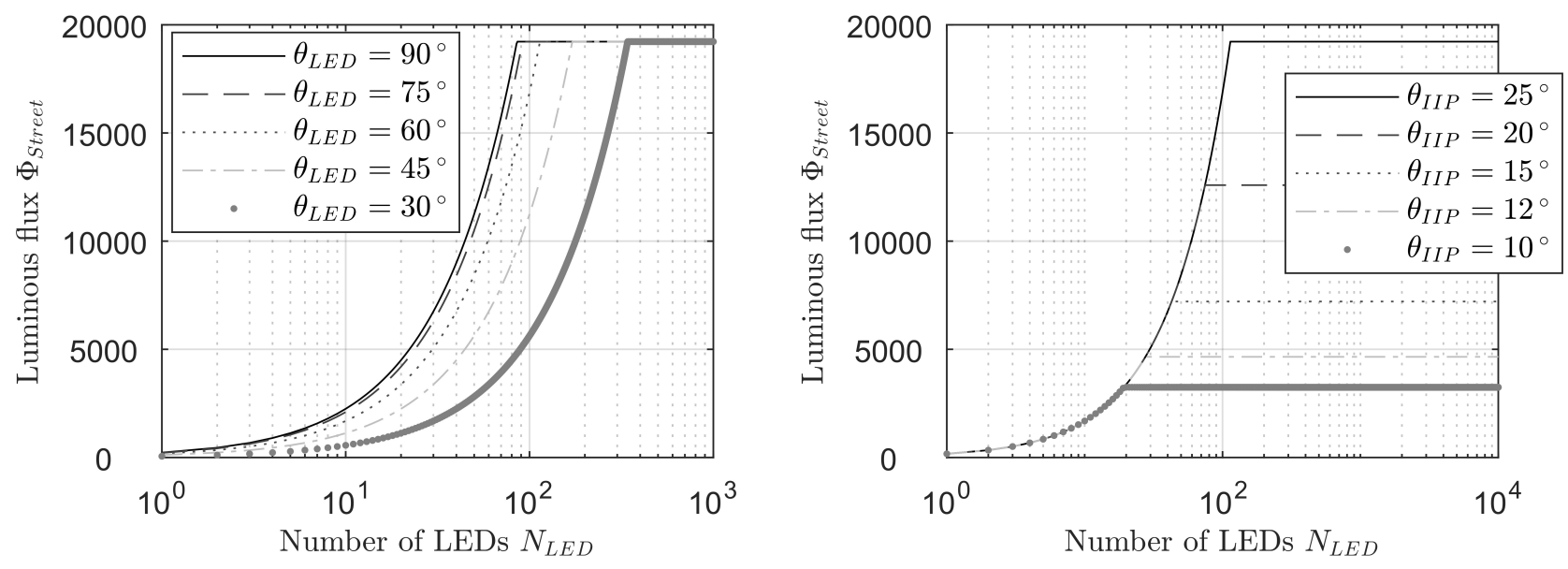

Figure 6. Luminous flux $\Phi_{\text {Street }}$ as a function of pixels $N_{L E D}$

Comparing both concepts by assuming the same impinging $\left(\theta_{S L M}=12^{\circ}\right)$ and acceptance angles $\left(\theta_{L E D}=60^{\circ}\right)$ it still concludes to

$$
\Phi_{\text {Street }, I I P}=4652.2 \mathrm{~lm} \text {, }
$$

which is over $609 \%$ more possible luminous flux than in a DMD-based concept. Last but not least, the values of the luminous flux as a function of LED numbers $N_{L E D}$ for an LED array-modulated concept is shown in figure 6 . As already mentioned, the ideal design point of $G_{F r a c}=1$ is identical for each acceptance angle. But this does not apply for the number of LEDs. This can be clearly seen in the left diagram for $\theta_{I I P}=25^{\circ}$. The lower the acceptance angle of the LED (and therefore the efficiency $\eta_{\theta}$ ), the more LEDs can be used for the light source. Concerning the impinging angle on the IIP this also concludes to a higher number of LEDs for higher impinging angles (see figure 6 right side).

\section{CONCLUSIONS AND OUTLOOK}

High-resolution vehicle headlamps are a future-orientated technology that can provide ideal light functions like projecting navigation information on the road or simultaneously dim small complex areas in the light distribution. The high resolution is achieved by using additive or subtractive spatial light modulators like an LED array or a DMD for image generation. By simplifying the light source emitter characteristics we showed an easy way 
to determine the maximum luminous flux, efficacy and resolution of the light source array for both concepts by using the hypothesis of Étendue conservation. Calculating the Fractional Étendue can predict the optimum between the three parameters at an early design stage and needs a simplification of the light source emitter characteristics. Therefore, the main influence parameter to increase the luminous flux is defined by the SLM Étendue while the main influence on efficacy is determined by the Étendue of the light source array. For an DMDbased system using the TI V-7000 DMD, we showed that the maximum luminous flux is limited to $763.65 \mathrm{~lm}$ which is not sufficient for using it as a headlamp. This can be compensated by either using a different type of LED with a higher luminous flux / WPE or by using a DMD which has an acceptance angle of $\pm 17^{\circ}$. The approach of an LED array-modulated headlamp offers a much higher potential with a luminous flux of $4652.2 \mathrm{~lm}$. Taking the other efficiencies such as optical efficiencies of the optics into account, this still provides a suitable solution. Nevertheless, the maximum amount of pixels still cannot compete to subtractive systems since the LED dimensions are too big limiting the maximum possible resolution to $N_{L E D}=114$ LEDs at $G_{F r a c}=1$. Reducing the light emitting surface to $0.125 \mathrm{~mm} \times 0.125 \mathrm{~mm}$ (similar to the $\mu \mathrm{AFS}^{9}$ ) can increase the number of pixels to approximately 27430 pixels which is a first step towards competitive systems.

\section{ACKNOWLEDGMENTS}

This project was funded by the Ministry of Science and Culture of Lower Saxony within the framework of the project Tailored Light.

\section{REFERENCES}

[1] Brunne, D. and Kalze, F.-J., "Outlook on high resolution pixel light," in [12th International Symposium on Automotive Lightning-ISAL 2017-Proceedings of the Conference: Volume 17], Proc. SPIE, 243 (2017).

[2] Kloppenburg, G., Wolf, A., and Lachmayer, R., "High-resolution vehicle headlamps: technologies and scanning prototype," Advanced Optical Technologies 5(2), 147-155 (2016).

[3] Jiao, J. and Wang, B., "High-efficiency reflector optics for led automotive forward lighting," in [Nonimaging Optics and Efficient Illumination Systems IV], 6670,66700M, International Society for Optics and Photonics (2007).

[4] Ley, P.-P., Held, M. P., and Lachmayer, R., "Konzepte für hochaufösende fahrzeugscheinwerfer unter berücksichtigung der étendue," DGaO-Proceedings 2017, ISSN: 1614-8436 (2017).

[5] Held, M. P. and Lachmayer, R., "Efficient conceptual design for led-based pixel light vehicle headlamps," in [Photonics, Devices and Systems VII], Proc. SPIE 10603, DOI: 10.1117/12.2292741 (2017).

[6] Instruments, T., "Dlp7000 dlp 0.7 xga 2x lvds type a dmd." Technical Datasheet (2017).

[7] Semiconductor, S., "High-power led for automotive sww0cs11s - wicop-c 1x1 3p white." Technical Datasheet (2017).

[8] Koshel, J., [Advanced nonimaging/illumination optics], Wiley-Blackwell, Oxford (2011).

[9] Grötsch, S., Brink, M., Fiederling, R., Liebetrau, T., Möllers, I., Moisel, J., Oppermann, H., and Pfeuffer, A., "uafs high resolution adb/afs solution," SAE Technical Paper 2016-01-1410, DOI: 10.4271/2016-01-1410 (2016). 